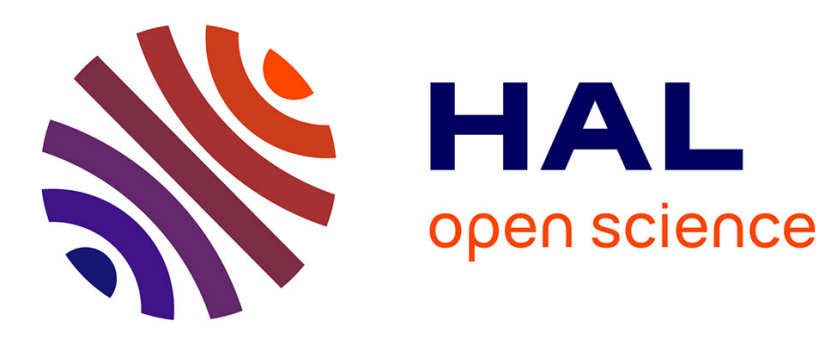

\title{
X-SFEM, a computational technique based on X-FEM to deal with random shapes
}

\author{
Anthony Nouy, Franck Schoefs, Nicolas Moës
}

\section{To cite this version:}

Anthony Nouy, Franck Schoefs, Nicolas Moës. X-SFEM, a computational technique based on XFEM to deal with random shapes. Revue Européenne de Mécanique Numérique/European Journal of Computational Mechanics, 2007, 16 (2), pp.277-293. 10.3166/remn.16.277-293 . hal-00368060

\section{HAL Id: hal-00368060 https://hal.science/hal-00368060}

Submitted on 13 Mar 2009

HAL is a multi-disciplinary open access archive for the deposit and dissemination of scientific research documents, whether they are published or not. The documents may come from teaching and research institutions in France or abroad, or from public or private research centers.
L'archive ouverte pluridisciplinaire HAL, est destinée au dépôt et à la diffusion de documents scientifiques de niveau recherche, publiés ou non, émanant des établissements d'enseignement et de recherche français ou étrangers, des laboratoires publics ou privés. 


\title{
X-SFEM, a computational technique based on X-FEM to deal with random shapes
}

\author{
Anthony Nouy — Franck Schoefs - Nicolas Moës \\ GeM, Institut de recherche en Génie civil et mécanique \\ Université de Nantes/UMR CNRS 6183/Ecole Centrale de Nantes \\ 2 rue de la Houssinière, BP 92208, F-44322 Nantes cedex 3 \\ anthony.nouy@univ-nantes.fr
}

\begin{abstract}
We propose a new method to deal with random geometries. It is an extension to the stochastic context of the eXtended Finite Element Method. This method lies on two majors points: the implicit description of geometry by the level set technique and the use of the partition of unity method for the enrichment of the finite element approximation space. This new technique leads by a direct calculus on a fixed finite element mesh to a solution which is explicit in terms of the basic random variables describing the geometry. We present here the basis of this approach and several examples to illustrate its performances.

RÉSUMÉ. Nous proposons une nouvelle méthode éléments finis stochastiques permettant de prendre en compte le caractère aléatoire de la géométrie dans le calcul de structures. Cette approche est basée sur la technique X-FEM, étendue au cadre stochastique. Elle repose sur deux points importants : la description fonctionnelle implicite de la géométrie par la technique des level sets et l'utilisation de la partition de l'unité pour l'enrichissement de l'espace d'approximation. Cette approche, qu'on pourrait baptiser naturellement eXtended Stochastic Finite Element Method (X-SFEM), permet d'obtenir par un calcul «direct » une solution explicite en fonction des variables aléatoires décrivant le caractère aléatoire de la géométrie. On présente ici les fondements de cette méthode et des exemples illustrent ses performances.

KEYWORDS: computational stochastic mechanics, X-FEM, level set, stochastic finite element, random geometry.

MOTS-CLÉS : calcul stochastique, X-FEM, level set, éléments finis stochastiques, géométrie aléatoire.
\end{abstract}

Preprint 


\section{Introduction}

A great challenge in the last few years has been to develop robust and efficient computational strategies to take into account uncertainties in structural analysis. Stochastic finite element methods (Ghanem et al., 1991; Ghanem, 1999; Deb et al., 2001; Matthies et al., 2005) have been recently proposed and offers a significant tool to deal with random material properties and boundary conditions. They lead to a very fine solution at the stochastic level which is explicit in terms of the random variables describing the uncertainties. Their efficiency, constantly improved by the use of ad hoc resolution techniques (Pellissetti et al., 2000; Keese et al., 2005; Nouy et al., 2005), allows one to use them in various fields of structural analysis such as design, reliability analysis... Dealing with uncertainties on applied loads or on material properties is a question relatively mastered within these methods. However, there is still no available efficient strategy to deal with uncertainties in the geometry although it could have a great interest in various applications: modeling of uncertainties in fabrication processes (size or position of borings, shape of a weld), of loss of matter due to corrosion, of random multi-phased materials. A traditional Monte-Carlo simulation could be performed to obtain statistics of the response. However, each event is associated with a new geometry and the use of classical finite element methods implies numerous remeshings and leads to prohibitive computational costs. To reduce the cost of remeshings, an alternative technique could consist in introducing variabilities on the position of the nodes of the finite element model. Nevertheless, this technique is limited to small variabilities of the geometry to avoid intolerable distorsion of the mesh and numerical instabilities. For large variabilities, this kind of techniques also need many remeshings.

Here, we propose a new stochastic finite element method which enables to deal with random geometry without remeshings. It is based on the extended finite element method (Moës et al., 1999; Sukumar et al., 2001), extended to the stochastic context. This method relies on two majors points: implicit description of the geometry by the level set technique (Sethian, 1999) and use of the partition of unity method (Melenk et al., 1996) for the enrichment of the finite element approximation space. The approach proposed here is a new intrusive technique which we call eXtended Stochastic Finite Element Method (X-SFEM). It leads by a direct calculus on a fixed finite element mesh to a solution which is explicit in terms of the basic random variables describing the geometry. A particular care is taken on the choice of approximation spaces. Notably, we show how the use of finite element approximation at the stochastic level can consequently improve the performances of this approach. In this article, we will focus on uncertainties on the shape of the domain. Enrichment by the partition of unity to model internal surfaces as cracks or material interfaces will be introduced in a subsequent paper. The method proposed here also offers a significant tool for parametric analysis which could be performed during the design stage of structures.

The plan of the paper is as follows: in Section 2, the problem involving random geometry is introduced mathematically. In Section 3, a probabilistic modeling of the geometry using the level set technique is presented. Approximation spaces are in- 
troduced in Section 4. The discretized problem, its construction and resolution are also presented in Section 4. Two examples will illustrate the capabilities of our approach in Section 5. We will emphasize in particular on the discretization choice at the stochastic level.

\section{Formulation of a stochastic problem with random geometry}

\subsection{Formulation of the continuous problem}

We will focus for simplicity on the case of a linear elliptic stochastic partial differential equation defined on a random domain. We denote by $(\Theta, \mathcal{B}, P)$ the probability space, where $\Theta$ is the set of outcomes, $\mathcal{B}$ the $\sigma$-algebra of events and $P$ the probability measure. The random geometry is defined by the mapping $\theta \in \Theta \rightarrow \Omega(\theta) \subset \mathbb{R}^{d}$. The stochastic problem is: find $u(\theta, x)$ which satisfies $P$-almost everywhere on $\Theta$,

$$
\begin{aligned}
& -\nabla \cdot(a(\theta, x) \nabla u(\theta, x))=f(\theta, x) \quad \text { on } \quad \Omega(\theta) \\
& u=0 \quad \text { on } \quad \Gamma_{1}(\theta) \quad n \cdot a \nabla u=g(\theta, x) \quad \text { on } \quad \Gamma_{2}(\theta)
\end{aligned}
$$

where $a$ is the diffusion coefficient, possibly random. $\Gamma_{1}$ is the part of $\partial \Omega(\theta)$ where Dirichlet conditions are imposed and $\Gamma_{2}(\theta)$ is the complementary part to $\Gamma_{1}$. In this article, we consider that $\Gamma_{1}$ is deterministic.

The variational formulation of the problem can be written: find $u \in \mathcal{V}_{0}=\{u \in$ $\left.\mathcal{V} ; u_{\mid \Theta \times \Gamma_{1}}=0\right\}$ such that

$$
\begin{aligned}
A(u, v) & =L(v) \quad \forall v \in \mathcal{V}_{0} \\
\text { with } \quad A(u, v) & =\int_{\Theta} \int_{\Omega(\theta)} a \nabla u \cdot \nabla v d x d P(\theta) \\
L(v) & =\int_{\Theta} \int_{\Omega(\theta)} f v d x d P(\theta)+\int_{\Theta} \int_{\Gamma_{2}(\theta)} g v d s d P(\theta)
\end{aligned}
$$

\subsection{Choice of ad hoc function spaces}

The question of the choice of an ad hoc function space $\mathcal{V}_{0}$ in order to prove existence and uniqueness of the solution of problem [2] has not been treated in the litterature to the knowledge of the authors. Although presented in the deterministic context, we can find in (Babuska et al., 2002; Babuska, 2003) some results on the effects of uncertainties in the domain on the solution of stochastic boundary value problems. Here, we introduce a mathematical framework which is adapted to the method proposed in this article.

Let us consider a domain $B$ such that $\overline{\bigcup_{\theta} \Omega(\theta)} \subset B$. We assume that $a$ is bounded and uniformly coercive. Under classical regularity assumptions on $a$ and the right 
hand side of [2], the solution $u$, for a fixed $\theta$, belongs to the space $H^{1}(\Omega(\theta))$. By prolongation, this space can be considered as a subspace of $H^{1}(B)$. Then, we will work on the following function space:

$$
\begin{aligned}
\mathcal{V} & =L^{2}\left(\Theta, d P ; H^{1}(B)\right) \\
& =\left\{v: \Theta \times B \rightarrow \mathbb{R} \mid \int_{\Theta}\|v(\theta, .)\|_{H^{1}(B)}^{2} d P(\theta)<\infty\right\}
\end{aligned}
$$

The bilinear and linear forms in problem [2] are then rewritten as follows:

$$
\begin{aligned}
& A(u, v)=\int_{\Theta} \int_{B} a \nabla u \cdot \nabla v I_{\Omega} d x d P(\theta) \\
& L(v)=\int_{\Theta} \int_{B} f v I_{\Omega} d x d P(\theta)+\int_{\Theta} \int_{\Gamma_{2}(\theta)} g v d s d P(\theta)
\end{aligned}
$$

where $I_{\Omega}(\theta, \cdot): B \rightarrow\{0,1\}$ denotes the characteristic function of $\Omega(\theta)$.

The bilinear form $A$ is bounded but only semi-coercive on $\mathcal{V}$. The kernel in $\mathcal{V}_{0}$ of $v \rightarrow A(v, v)$ is a set of functions whose support is a subset of what we can call the "non-physical" domain $\mathcal{N}=\{(\theta, x) \in \Theta \times B ; x \notin \Omega(\theta)\}$. Problem [2] have an infinite number of solutions which differ from one another by functions in this kernel. Let us briefly prove this statement. Let $u_{1}$ and $u_{2}$ be two solutions of [2] and $w=u_{1}-u_{2} \in \mathcal{V}_{0}$. We have $A(w, w)=0$. Then, $a \nabla w \cdot \nabla w I_{\Omega}=0$ almost surely on $\Theta \times B$. Let us denote by $\mathcal{N}^{c}=\{(\theta, x) \in \Theta \times B ; x \in \Omega(\theta)\}$ the "physical" domain, which is the complementary set of $\mathcal{N}$. We have $I_{\Omega}(\theta, x)=1$ on $\mathcal{N}^{c}$ and 0 on $\mathcal{N}$ (i.e. $I_{\Omega}$ is the characteristic function of $\mathcal{N}^{c}$ ). Due to positivity properties of $a$, we have $\nabla w=0$ almost surely on $\mathcal{N}^{c}$ and $w_{\mid \Theta \times \Gamma_{1}}=0$. We then conclude that $w=0$ almost surely on $\mathcal{N}^{c}$. The "physical" part of the solution, i.e. its restriction to $\mathcal{N}^{c}$, is then unique. And this is the part of the solution we are interested in ! Prolongation of the solution in $H^{1}(B)$ in the stochastic context will not have any physical meaning. For example, if we consider a point $x \in B$ such that $P(x \in \Omega(\theta))<1$, the quantity $u(\theta, x)$ will make sense only for outcomes of domain which include $x$. A good postprocessing will have to avoid such quantities of interest. We will rather be interested in quantities defined on a well defined zone (such as mean displacement on a part of the boundary... ) or on implicit quantities such as the maximum stress.

\section{Representing random geometry by the level set technique}

\subsection{Definition and approximation of level sets}

In the deterministic context, the level set technique (Sethian, 1999) consists in representing a surface by the zero level set of a function. We commonly use for this function the signed function to the surface. This function, denoted by $\phi(x)$, is gen- 
erally interpolated at nodes of a fixed finite element mesh which does not conform to the physical surface:

$$
\phi(x)=\sum_{i} N_{i}(x) \phi_{i}=\mathbf{N}(x) \phi
$$

where $N_{i}$ are the classical finite element shape functions and where $\mathbf{N}=$ $\left[N_{1}, \ldots, N_{N}\right]$, with $N$ the number of nodes. In the probabilistic context, this function simply becomes a stochastic field $\phi(\theta, x)$, where $\theta$ denotes the outcome. For a given outcome $\theta$, the zero level set of $\phi(\theta, x)$ represent the corresponding outcome of the random surface. In the same way, this field will be interpolated at nodes of a fixed finite element mesh and will then be described by a vector of random variables $\phi(\theta)$. The question is: how to build this stochastic field? In all cases, for the resolution of the stochastic problem, we will try to reduce the source of randomness to a small number of independent random variables $\boldsymbol{\xi}(\theta)$. The level set function will then be defined by

$$
\phi(\boldsymbol{\xi}(\theta), x)=\mathbf{N}(x) \phi(\boldsymbol{\xi}(\theta))
$$

First, we can chose an a priori representation of the geometry parameterized in terms of a set of random variables, whose probabilistic laws can be identified from experimental data. Let us take for example the case of a circular hole with random center $c(\theta)$, whose position depends on two random variables in $2 D$, and with random radius $R(\theta)$. In this case, the level set can be explicitly written as follows: $\phi(\theta, x)=R(\theta)-\|x-c(\theta)\|$. Explicit expressions of level sets exist for several classical geometrical forms (ellipses, polygones...) (Sukumar et al., 2001).

If the geometry can be written as a function of a stochastic field, a discretization step of this field and a systematic construction of the corresponding level set will be necessary. These points will be detailed in a subsequent paper.

\subsection{Representation of an external random geometry}

When the domain $\Omega$ is random, we introduce a level set function $\phi(\theta, x)$ whose iso-zero surface represents the boundary $\partial \Omega(\theta)$. We suppose that $\phi(\theta, x)$ is negative in $\Omega(\theta)$ and positive in the complementary part. Denoting by $\mathcal{H}$ the Heaviside function, the characteristic function of the domain can simply be written:

$$
I_{\Omega}(\theta, x)=\mathcal{H}(-\phi(\theta, x))
$$

\section{Discretization of the problem}

In this part, we introduce the discretization of problem [2] at the spatial and stochastic levels. In this article, we only consider random shapes. It is a particular case of X-SFEM for which no enrichment is needed (see (Daux et al., 2000) for the deterministic case). 


\subsection{Preliminaries and notations}

We assume that the probabilistic modeling stage has allowed us to represent the source of randomness with a finite number $m$ of independent random variables $\boldsymbol{\xi}(\theta)=$ $\left(\xi_{1}(\theta), \ldots, \xi_{m}(\theta)\right)$. A random variable $v(\theta)$ can then be expressed in terms of $\boldsymbol{\xi}$ : $v(\theta) \equiv v(\boldsymbol{\xi}(\theta))$. We then work in the image probability space $\boldsymbol{\Theta}=\boldsymbol{\xi}(\Theta) \subset \mathbb{R}^{m}$ endowed with probability measure $P_{\boldsymbol{\xi}}$ defined for Borel sets $b \in \mathcal{B}_{\mathbb{R}^{m}}$ by $P_{\boldsymbol{\xi}}(b)=$ $P\left(\boldsymbol{\xi}^{-1}(b)\right)$. We denote by $E($.$) the mathematical expectation, defined by$

$$
E(v)=\int_{\Theta} v(\theta) d P(\theta)=\int_{\Theta} v(\mathbf{x}) d P_{\boldsymbol{\xi}}(\mathbf{x})
$$

\subsection{Choice of approximation spaces}

We first notice that $\mathcal{V}$, defined in [3], is the tensor product of the spaces $\mathcal{S}=L^{2}(\boldsymbol{\Theta})$ and $\mathcal{U}=H^{1}(B)$. We then choose a tensor product approximation. The domain $B$ is discretized by a mesh $\mathcal{T}_{h}$ yielding a finite element approximation space $\mathcal{U}_{h} \subset \mathcal{U}$. The set of shape functions is denoted by $\left\{N_{i}\right\}_{i \in I}$, where $I$ denotes the set of nodes. At the stochastic level, we introduce $\left\{H_{\alpha}(\boldsymbol{\xi})\right\}_{\alpha \in \mathcal{I}_{P}}$, a basis of $P$ functions of $\mathcal{S}_{P} \subset \mathcal{S}$. The approximate solution, denoted by $u_{h, P}$, will then be searched in the space

$$
\begin{aligned}
\mathcal{V}_{h, P}=\mathcal{S}_{P} \otimes \mathcal{U}_{h} & =\left\{\sum_{\alpha \in \mathcal{I}_{P}} \sum_{i \in I} N_{i}(x) H_{\alpha}(\boldsymbol{\xi}) u_{i, \alpha}, u_{i, \alpha} \in \mathbb{R}\right\} \\
& =\left\{\sum_{\alpha \in \mathcal{I}_{P}} \mathbf{N}(x) \mathbf{u}_{\alpha} H_{\alpha}(\boldsymbol{\xi}), \mathbf{u}_{\alpha} \in \mathbb{R}^{N}\right\}
\end{aligned}
$$

where $\mathbf{N}=\left[N_{1}, \ldots, N_{n}\right], n$ being the number of nodes in the mesh.

Several choices have been proposed for building approximation basis of $L^{2}(\boldsymbol{\Theta})$. Spectral approaches as polynomial chaos (Wiener, 1938; Ghanem et al., 1991) or generalized polynomial chaos (Xiu et al., 2002) use orthogonal polynomial basis and exhibit exponential convergence rates (Xiu et al., 2002) in the case of regular solutions. For the case of less regular solutions, other approximation techniques were introduced (Wiener-Haar chaos (Le Maître et al., 2004), hp finite elements (Deb et al., 2001)).

In practice, the basis functions will be taken orthonormal: $E\left(H_{\alpha} H_{\beta}\right)=1$ if $\alpha=\beta$ and 0 otherwise.

\subsubsection{Polynomial chaos}

We note that $\mathcal{S}$ is the product space $\mathcal{S}^{1} \otimes \ldots \otimes \mathcal{S}^{m}$, where $\mathcal{S}^{i}=L^{2}\left(\Theta^{i}, \mathcal{B}^{i}, d P_{\xi_{i}}\right)$. The polynomial chaos discretization consists in choosing for $\mathcal{S}_{P}$ a space of multidimensional polynomials $H_{\alpha}=h_{\alpha_{1}}^{1}\left(x_{1}\right) \ldots h_{\alpha_{n}}^{n}\left(x_{n}\right)$, where $h_{\alpha_{i}}^{i} \in \mathcal{S}_{i}$ and where $\alpha=\left(\alpha_{1}, \ldots, \alpha_{m}\right)$ denotes a multi-index of $\mathbb{N}^{m}$. Polynomials are chosen orthogonal with respect to the scalar product of $\mathcal{S}=L^{2}\left(\boldsymbol{\Theta}, d P_{\boldsymbol{\xi}}\right)$, defined by $(u, v)=E(u v)$. The method initially proposed by (Ghanem et al., 1991) consists in working with a 
set $\boldsymbol{\xi}$ of independent Gaussian variables. In this case, $h_{j}^{i}$ denotes Hermite polynomials which are the orthogonal polynomials with respect to Gaussian measure. The same basis functions are used for all stochastic dimensions. Classically, we choose for $\mathcal{S}_{P}$ the polynomial chaos of order $p$ in $m$ variables, defined with multi-indices: $\mathcal{I}_{P}=\left\{\alpha \in \mathbb{N}^{m} ;|\alpha|=\sum_{i=1}^{m} \alpha_{i} \leqslant p\right\}$. The number of basis functions of $\mathcal{S}_{P}$ is $P=\frac{(m+p) !}{p ! m !}$

The generalized polynomial chaos method (Xiu et al., 2002) uses orthogonal polynomials with respect to measures $d P_{\xi_{i}}$ for $h_{j}^{i}$. In the case of uniform, beta or gamma random variables, we use respectively Legendre, Jacobi and Laguerre polynomial. For arbitrary measure and possibly dependent variables, it is also possible to introduce generalized chaos decompositions (Soize et al., 2004).

\subsubsection{Finite elements at the stochastic level}

Finite element approximation at the stochastic level (Deb et al., 2001; Babuska et al., 2005) consists in choosing for $\mathcal{S}_{P}$ a space of piecewise polynomial functions (discontinuous) on a mesh of $\Theta$. This type of approximation allows one to use tools which are classical in the finite element context such as a posteriori error estimation, adaptative refinement...

Here, we suppose that $\Theta=\Theta^{1} \times \ldots \times \Theta^{m}$ is a bounded open set of $\mathbb{R}^{m}$. On each stochastic dimension, we introduce a mesh $\mathcal{T}_{k}^{i}$ of $n_{i}$ elements of $\Theta^{i}$. We denote by $\mathcal{T}_{k}=\mathcal{T}_{k}^{1} \times \ldots \times \mathcal{T}_{k}^{m}$ the corresponding rectangular mesh of $\mathbb{R}^{m}$. The restriction of a function $H_{\alpha}(\mathbf{x})$ of $\mathcal{S}_{P}$ to an element $\tau$ of $\mathcal{T}_{k}$ satisfies

$$
H_{\alpha \mid \tau} \in \operatorname{span}\left(\prod_{i=1}^{m} x_{i}^{q_{i}}, q_{i} \in \mathbb{N} \text { and } q_{i} \leqslant p_{i}\right)
$$

where $p_{i}$ is the approximation degree on $\Theta^{i}$. Here, we choose for $\mathcal{I}_{P}$ the set: $\mathcal{I}_{P}=$ $\left\{\alpha=\left(\alpha_{1}, \ldots \alpha_{m}\right) \in\left(\mathbb{N}^{2}\right)^{m}, \alpha_{i}=\left(j_{i}, q_{i}\right), 1 \leqslant j_{i} \leqslant n_{i}, 0 \leqslant q_{i} \leqslant p_{i}\right\}$. The number of basis functions of $\mathcal{S}_{P}$ is here $P=\prod_{i=1}^{m} n_{i}\left(p_{i}+1\right)$. We recall that the basis functions are orthonormalized.

\subsection{Definition of the approximate solution}

We introduce $\mathcal{V}_{h, P, 0}=\mathcal{V}_{h, P} \cap \mathcal{V}_{0}$. The Galerkin approximation $u_{h, P} \in \mathcal{V}_{h, P, 0}$ of [2] is defined by

$$
A\left(u_{h, P}, v_{h, P}\right)=L\left(v_{h, P}\right), \forall v_{h, P} \in \mathcal{V}_{h, P, 0}
$$

To avoid singularity in the discretized problem [12], it is necessary to suppress functions of $\mathcal{V}_{h, P}$ lying in the kernel of $v \rightarrow A(v, v)$. In particular, that leads to suppress degrees of freedom associated with the set of nodes $I_{\text {out }}$ whose support is $P_{\xi}$-almost surely outside the physical domain. Denoting by $\omega_{i}$ the support of shape 
function $N_{i}$, i.e. the union of elements having node $i$ as one of their vertices, the set $I_{\text {out }}$ can be written:

$$
I_{\text {out }}=\left\{i \in I ; P_{\boldsymbol{\xi}}\left(\omega_{i} \cap \Omega(\boldsymbol{\xi})=\emptyset\right)=1\right\}=\left\{i \in I ; \omega_{i} \subset \Omega_{0}\right\}
$$

where $\Omega_{o}$ is the biggest open set almost surely outside $\Omega$, i.e. such that $P_{\xi}\left(\Omega_{0} \cap\right.$ $\Omega(\xi)=\emptyset)=1$. Figure 1 illustrate this definition in the case of a circular hole with random radius.

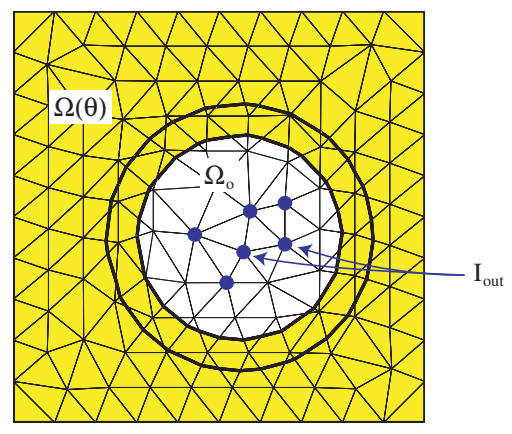

Figure 1. Circular hole with random radius (bounded): definition of the node set $I_{\text {out }}$. $\Omega_{o}$ is the biggest open set such that $P_{\boldsymbol{\xi}}\left(\Omega_{o} \cap \Omega(\boldsymbol{\xi})=\emptyset\right)=1$

Suppressing these degrees of freedom is only a necessary condition for the nonsingularity of the discretized problem [12]. In general, we will then have to solve a semi-definite system of equations for which we will search a particular solution (see Section 2.2).

\subsection{The discretized problem and its resolution}

Discretized problem [12] can also be written: find $u \in \mathcal{V}_{h, P, 0}$ such that $\forall v \in$ $\mathcal{V}_{h, P, 0}$

$$
\begin{aligned}
& A\left(\sum_{\alpha \in \mathcal{I}_{P}} \mathbf{N}(x) \mathbf{v}_{\alpha} H_{\alpha}(\boldsymbol{\xi}), \sum_{\beta \in \mathcal{I}_{P}} \mathbf{N}(x) \mathbf{u}_{\beta} H_{\beta}(\boldsymbol{\xi})\right)=L\left(\sum_{\alpha \in \mathcal{I}_{P}} \mathbf{N}(x) \mathbf{v}_{\alpha} H_{\alpha}(\boldsymbol{\xi})\right) \\
\Longrightarrow & \sum_{\alpha, \beta \in \mathcal{I}_{P}} \mathbf{v}_{\alpha}^{T} E\left(\mathbf{K} H_{\alpha} H_{\beta}\right) \mathbf{u}_{\beta}=\sum_{\alpha \in \mathcal{I}_{P}} \mathbf{v}_{\alpha}^{T} E\left(\mathbf{f} H_{\alpha}\right)
\end{aligned}
$$

where the random matrix $\mathbf{K}$ and the random vector $\mathbf{f}$ are defined by:

$$
\begin{aligned}
& \mathbf{K}(\boldsymbol{\xi})=\int_{B} a(\boldsymbol{\xi}, x) \nabla \mathbf{N}^{T} \nabla \mathbf{N} \mathcal{H}(-\phi(\boldsymbol{\xi}, x)) d x \\
& \mathbf{f}(\boldsymbol{\xi})=\int_{B} \mathbf{N}^{T} f \mathcal{H}(-\phi(\boldsymbol{\xi}, x)) d x+\int_{\Gamma_{2}(\boldsymbol{\xi})} \mathbf{N}^{T} g d s
\end{aligned}
$$


Let $\mathbf{K}_{\alpha \beta}=E\left(\mathbf{K} H_{\alpha} H_{\beta}\right)$ and $\mathbf{f}_{\alpha}=E\left(\mathbf{f} H_{\alpha}\right)$. System [14] can then be rewritten:

$$
\left(\begin{array}{cccc}
\mathbf{K}_{\alpha_{1} \alpha_{1}} & \mathbf{K}_{\alpha_{1} \alpha_{2}} & \ldots & \mathbf{K}_{\alpha_{1} \alpha_{P}} \\
\mathbf{K}_{\alpha_{2} \alpha_{1}} & \ddots & & \vdots \\
\vdots & & \ddots & \vdots \\
\mathbf{K}_{\alpha_{P} \alpha_{1}} & \ldots & \ldots & \mathbf{K}_{\alpha_{P} \alpha_{P}}
\end{array}\right)\left(\begin{array}{c}
\mathbf{u}_{\alpha_{1}} \\
\mathbf{u}_{\alpha_{2}} \\
\vdots \\
\mathbf{u}_{\alpha_{P}}
\end{array}\right)=\left(\begin{array}{c}
\mathbf{f}_{\alpha_{1}} \\
\mathbf{f}_{\alpha_{2}} \\
\vdots \\
\mathbf{f}_{\alpha_{P}}
\end{array}\right)
$$

In practice, system [17] can not be solved by a direct resolution technique. Indeed, the memory storage and computational costs of assembling and solving this huge system become prohibitive for common engineering problems. To avoid assembling and to take part of the sparsity of this system, we classically use Krylov-type iterative resolution techniques (Ghanem et al., 1996; Pellissetti et al., 2000; Keese et al., 2005). An alternative approach has been proposed in (Nouy et al., 2005), which consists in searching an optimal spectral decomposition of the solution. An ad hoc construction of this decomposition allows one to transform the problem into the resolution of a few uncoupled deterministic spatial finite element problems and stochastic equations. This approach reduces drastically computational and storage costs.

\subsection{Computing matrices and vectors of the discretized problem}

For solving [17], matrices $\mathbf{K}_{\alpha \beta}=E\left(\mathbf{K} H_{\alpha} H_{\beta}\right)$ have to be computed. Computing these matrices independently would lead to prohibitive costs. In practice, we first decompose $\mathbf{K}$ on an ortho-normal basis of $\mathcal{S}_{P^{\prime}} \subset \mathcal{S}$ :

$$
\mathbf{K}(\boldsymbol{\xi})=\sum_{\alpha \in \mathcal{I}_{P^{\prime}}} \mathbf{K}_{\alpha} H_{\alpha}(\boldsymbol{\xi}) \text { with } \quad \mathbf{K}_{\alpha}=E\left(\mathbf{K} H_{\alpha}\right)
$$

This decomposition being performed, we simply have:

$$
E\left(\mathbf{K} H_{\alpha} H_{\beta}\right)=\sum_{\gamma \in \mathcal{I}_{P^{\prime}}} \mathbf{K}_{\gamma} E\left(H_{\gamma} H_{\alpha} H_{\beta}\right)
$$

REMARK. - Here, $\mathcal{S}_{P^{\prime}}$ will be chosen different from $\mathcal{S}_{P}$, the approximation space of the solution. Indeed, in the case where $\mathcal{S}_{P}$ is a space of orthogonal (piecewise) polynomials of degree less than $p$ (for polynomial chaos or finite element approximation at the stochastic level), the value of $\mathbf{K}_{\alpha \beta}$ will be exact if $\mathcal{S}_{P^{\prime}}$ contains orthogonal polynomials of degree less than $2 p$. With this choice, the approximation made on $\mathbf{K}$ will have no influence on the solution of the discretized problem [14] (see proof in (Matthies et al., 2005)).

In the decomposition [18], $\mathbf{K}_{\alpha}$ terms are calculated by Gauss-quadrature:

$$
\mathbf{K}_{\alpha}=E\left(\mathbf{K} H_{\alpha}\right)=\sum_{k=1}^{n_{g}} \mathbf{K}\left(\boldsymbol{\xi}_{k}\right) H_{\alpha}\left(\boldsymbol{\xi}_{k}\right) \omega_{k}
$$


where $n_{k}$ is the number of gauss points, $\omega_{k}$ the weights and $\boldsymbol{\xi}_{k}$ the integration points associated to the measure $d P_{\boldsymbol{\xi}}$. The calculation of all the matrices $\mathbf{K}_{\alpha}$ then only requires the assembling of $n_{g}$ matrices

$$
\mathbf{K}\left(\boldsymbol{\xi}_{k}\right)=\int_{B} a\left(\boldsymbol{\xi}_{k}, x\right) \nabla \mathbf{N}(x)^{T} \nabla \mathbf{N}(x) \mathcal{H}\left(-\phi\left(\boldsymbol{\xi}_{k}, x\right)\right) d x
$$

$\phi\left(\boldsymbol{\xi}_{k}, x\right)$ is a deterministic level set corresponding to an outcome of the geometry and, therefore, the calculation of matrices $\mathbf{K}\left(\boldsymbol{\xi}_{k}\right)$ is a classical calculation within the context of deterministic X-FEM method. In practice, to perform spatial integration, we use a classical Gauss-quadrature on elements which are not cut by a level set. Elements which are cut by a level set are first split into sub-elements on which we perform a classical Gauss-quadrature (Moës et al., 1999; Dolbow et al., 2000).

Vectors $\mathbf{f}_{\alpha}=E\left(\mathbf{f} H_{\alpha}\right)$ of the right hand side of [17] are calculated by a similar numerical integration. Their calculation is still classical within the X-FEM method.

\subsection{Back to the choice of stochastic discretization}

Parametrized problem equivalent to problem [14] can be written: $\mathbf{K}(\boldsymbol{\xi}) \mathbf{u}(\boldsymbol{\xi})=$ $\mathbf{f}(\boldsymbol{\xi}), \boldsymbol{\xi} \in \boldsymbol{\Theta}$. In fact, matrices and vectors of this problem are only piecewise regular on $\Theta$. This will be illustrated in example 1. Therefore, the use of a spectral discretization at the stochastic level such as polynomial chaos is not optimal. A better choice consists in using at the stochastic level a finite element discretization on a mesh $\mathcal{T}_{k}$ of $\Theta$ such that matrices and vectors are regular on the elements of $\mathcal{T}_{k}$. We use for building this mesh an automatic procedure which will be presented in a forecoming paper. In the case where X-FEM method gives an exact solution to the associated deterministic problem, this choice of approximation can allow one to obtain the exact solution of the stochastic problem [2]. When using classical polynomial chaos approximation, this meshing procedure of $\Theta$ is also needed to well integrate matrices and vectors of the discretized problem [17].

\section{Examples}

\subsection{Example 1: traction of a beam with random length}

We consider an homogeneous beam in traction laying in the random domain $\Omega=$ ] $0, \xi$, where $\xi$ is a uniform random variable: $\xi=U(0.25,0.75)$. We take $B=] 0,1[$ as a prolongation of the random domain. The traction modulus is taken equal to 1 . The beam is embedded at $x=0$ and is submitted to a uniform and unitary body load. The problem can be written:

$$
\left.\frac{d^{2} u}{d x^{2}}(\xi, x)+1=0 \quad \text { for } x \in\right] 0, \xi\left[, \quad u(\xi, 0)=0, \quad \frac{d u}{d x}(\xi, \xi)=0\right.
$$

An exact solution $u$ to this problem exists: $u(\xi, x)=x\left(\xi-\frac{x}{2}\right)$ on $] 0, \xi[$. 
Domain $B$ is meshed with $n$ elements of length $h=\frac{1}{n}$. The used level set set is $\phi(\xi, x)=x-\xi$. At the spatial level, we use $P^{1}$ finite elements. Figure 2 shows a 10 elements mesh of $B$, possible outcomes of domain $\Omega(\xi)$ and shows the node set $I_{\text {out }}$ defined in [13] for which we must suppress the associated degrees of freedom.

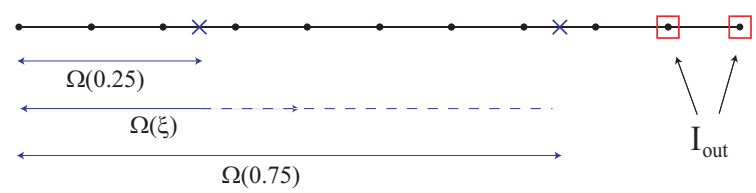

Figure 2. Example 1: 10 elements mesh of $B=] 0,1[$. Outcomes of physical domain $\Omega(\xi)$ and node set $I_{\text {out }}$ whose support is almost surely outside $\Omega(\xi)$

We first consider a generalized polynomial chaos approximation of degree $p$ at the stochastic level (Section 4.2.1). Basis functions of $\mathcal{S}_{P}$ are Legendre polynomials. To compare the solution $u_{h, P}$ of problem [12] to the exact solution $u$, we introduce two error indicators:

$$
\begin{aligned}
& e_{h, P}=\left\|u_{h, P}-u\right\|_{L^{2}\left(\boldsymbol{\Theta} ; L^{2}(\Omega(\xi))\right.}=E\left(\int_{\Omega(\xi)}\left(u_{h, P}(\xi, x)-u(\xi, x)\right)^{2} d x\right)^{1 / 2} \\
& \tilde{e}_{h, P}=\left\|u_{h, P}(\xi, \xi)-u(\xi, \xi)\right\|_{L^{2}(\boldsymbol{\Theta})}=E\left(\left(u_{h, P}(\xi, \xi)-u(\xi, \xi)\right)^{2}\right)^{1 / 2}
\end{aligned}
$$

The first indicator is a true error indicator. Indeed, when $e_{h, P} \rightarrow 0$, the physical part of the approximate solution (see Section 2.2 for this definition) converges toward the physical part of the exact solution. The second indicator represent the error which is made on a quantity of interest, namely the displacement at $x=\xi$ (the right extremity of the random domain). Table 1 shows the evolution of these errors with respect to the degree $(p)$ and mesh size $(h)$.

We can observe a good $h$ convergence of the approximation for both indicators, independent of the degree $p$ (convergence in $O\left(h^{2}\right)$ for $e_{h, P}$ and $O(h)$ for $\tilde{e}_{h, P}$ ). Since the exact solution $u(\xi, x)$ and the exact quantity of interest $u(\xi, \xi)$ are respectively polynomials of degree 1 and 2 in $\xi$, it is natural to converge with $h$ towards the exact solution, whatever the degree $p \geqslant 2$. We obtain a good approximation, especially for the quantity of interest, even with coarse meshes and a low degree of approximation at the stochastic level. We know that within the deterministic context, $\mathrm{X}$-FEM technique leads to an approximate solution $u_{h}(x)$ which gives an exact quantity of interest $u_{h}(\xi)$, whatever the mesh size $h$. We recover this property within the stochastic context for the coarser mesh $(h=1)$, for which we obtain an "exact" numerical solution.

REMARK. - For $h=1$, the fact that the error increases with the degree $p$ comes from round-off errors which become significant when we deal with higher order polynomials. For our purpose, we can then consider that these values of errors are approximately zero in a numerical sense. 
Table 1. Example 1: generalized chaos approximation. Error indicators $e_{h, P}^{P C}$ and $\tilde{e}_{h, P}^{P C}$ with respect to the degree $p$ of the chaos and the mesh size $h$

\begin{tabular}{|c|c|c|c|c|}
\hline$e_{h, P}^{P C}$ & $p=2$ & $p=4$ & $p=6$ & $p=8$ \\
\hline$h=1.0$ & $2.2210^{-2}$ & $2.2210^{-2}$ & $2.2210^{-2}$ & $2.2210^{-2}$ \\
$h=0.5$ & $1.3210^{-2}$ & $1.3210^{-2}$ & $1.3210^{-2}$ & $1.3210^{-2}$ \\
$h=0.2$ & $2.4210^{-3}$ & $2.3810^{-3}$ & $2.3810^{-3}$ & $2.3810^{-3}$ \\
$h=0.1$ & $6.7210^{-4}$ & $6.4310^{-4}$ & $6.3910^{-4}$ & $6.4010^{-4}$ \\
\hline \hline$\tilde{e}_{h, P}^{P C}$ & $p=2$ & $p=4$ & $p=6$ & $p=8$ \\
\hline$h=1.0$ & $9.7910^{-15}$ & $2.5510^{-14}$ & $7.5610^{-13}$ & $1.6110^{-12}$ \\
$h=0.5$ & $2.3410^{-3}$ & $1.3510^{-3}$ & $9.6610^{-4}$ & $7.5610^{-4}$ \\
$h=0.2$ & $1.2210^{-3}$ & $5.7610^{-4}$ & $3.8210^{-4}$ & $2.8710^{-4}$ \\
$h=0.1$ & $5.7610^{-4}$ & $2.6310^{-4}$ & $1.9110^{-4}$ & $1.4510^{-4}$ \\
\hline
\end{tabular}

However, we could have expected to obtain the exact quantity of interest for every mesh sizes. The fact that we do not obtain this property for more than 1 element is due to a non optimal choice of the approximation at the stochastic level. To better understand this, we consider the corresponding parametrized problem $\mathbf{K}(\xi) \mathbf{u}(\xi)=$ $\mathbf{f}(\xi)$ with $\xi \in] 0.25,0.75$ [ for a mesh with 2 elements. Figure 3 shows the evolution of components of $\mathbf{K}, \mathbf{f}$ and $\mathbf{u}$ with respect to $\xi$.
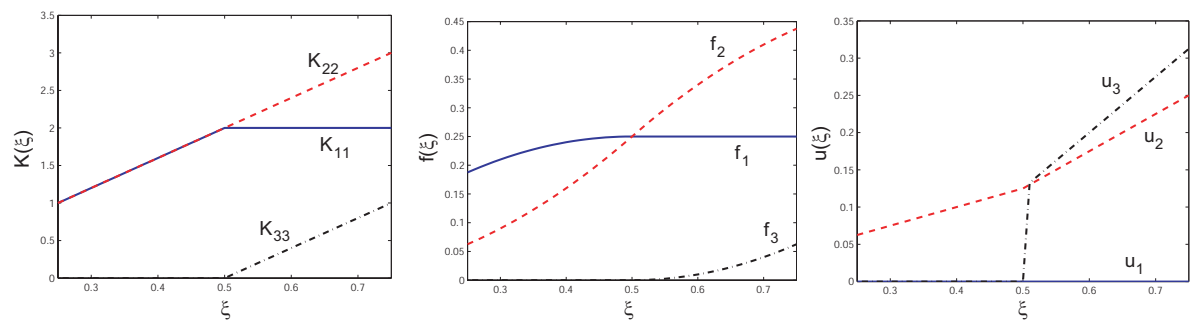

Figure 3. Components of $\mathbf{K}(\xi), \mathbf{f}(\xi)$ and $\mathbf{u}(\xi), \xi \in] 0.25,0.75$, for a mesh with 2 elements of length $\frac{1}{2}$. Indices correspond to node numbers, numbered from left to right

We note that these components are continuous piecewise polynomials, with a discontinuity point in their derivative located at $\xi=0.5$. This point corresponds to the outcome for which the extremity of the beam is located at the middle node. For example, let us prove this for $K_{11}(\xi)$. The support of shape function $N_{1}$ is the first element. On this element, $\frac{\partial N_{1}}{\partial x}=-2$. Then,

$$
K_{11}=4 \int_{0}^{0.5} \mathcal{H}(-\phi(\xi, x)) d x=4 \int_{0}^{\min (0.5, \xi)} d x=\left\{\begin{array}{l}
2 \text { for } \xi>0.5 \\
4 \xi \text { for } \xi<0.5
\end{array}\right.
$$


For $n$ elements, we can also easily show that discontinuity points in the derivatives appear for values of $\xi$ for which the extremity of the domain is located on a node. The use of spectral approximation is unadapted to represent non regular solutions and leads in this case to a bad $p$ convergence of the approximation at the stochastic level (for a fixed mesh size $h$ ).

Following these remarks, we propose to use at the stochastic level finite elements of degree $p$ (see Section 4.2.2) with a mesh $\Theta$ which coincides with the non-regular points. Of course, this mesh depends on the spatial mesh of $B$. Table 2 shows the evolution of error indicators with respect to degree $p$ and mesh size $h$. We observe that with an adaptive choice of the stochastic basis, we obtain the "exact" value (in the numerical sense) for the quantity of interest for all mesh sizes. The fact that the error increases while $h$ decreases or $p$ increases is due to round-off errors (see remark above). However, for fixed degree of approximation $p$ at the stochastic level, the global convergence is still in $O\left(h^{2}\right)$ : the values taken by indicators $e_{h, P}^{F E}$ (Table 2) and $e_{h, P}^{P C}$ (Table 1) are quite the same. In fact, non-regularities at the stochastic level which was explained above become smoother while the mesh size $h$ decrease. We can then expect that for small mesh size, polynomial chaos approximation gives satisfactory results.

Table 2. Example 1: finite element approximation of degree $p$ at the stochastic level. The mesh of $\Theta$ is adapted to the one of $B$. Error indicators $e_{h, P}^{F E}$ and $\tilde{e}_{h, P}^{F E}$ with respect to $p$ and $h$

\begin{tabular}{|c|c|c|c|c|}
\hline$e_{h, P}^{F E}$ & $p=2$ & $p=4$ & $p=6$ & $p=8$ \\
\hline$h=1.0$ & $2.2210^{-2}$ & $2.2210^{-2}$ & $2.2210^{-2}$ & $2.2210^{-2}$ \\
$h=0.5$ & $1.3210^{-2}$ & $1.3210^{-2}$ & $1.3210^{-2}$ & $1.3210^{-2}$ \\
$h=0.2$ & $2.3910^{-3}$ & $2.3910^{-3}$ & $2.3910^{-3}$ & $2.3910^{-3}$ \\
$h=0.1$ & $6.4410^{-4}$ & $6.4410^{-4}$ & $6.4410^{-4}$ & $6.4410^{-4}$ \\
\hline \hline$\tilde{e}_{h, P}^{F E}$ & $p=2$ & $p=4$ & $p=6$ & $p=8$ \\
\hline$h=1.0$ & $9.7910^{-15}$ & $2.5510^{-14}$ & $7.5610^{-13}$ & $1.6110^{-12}$ \\
$h=0.5$ & $8.3610^{-15}$ & $2.7910^{-14}$ & $9.2510^{-13}$ & $1.6410^{-12}$ \\
$h=0.2$ & $7.8210^{-15}$ & $3.5710^{-14}$ & $9.9410^{-13}$ & $7.5210^{-12}$ \\
$h=0.1$ & $7.8810^{-15}$ & $3.7210^{-14}$ & $1.0310^{-12}$ & $5.6210^{-12}$ \\
\hline
\end{tabular}

\subsection{Example 2: plate with a random hole in tension}

We consider the problem of a plate with a traction-free circular hole submitted to uniaxial tension. The plate lies in a square domain $] 0,3[\times] 0,3[$. The center of the hole is located at $(1.5,1.5)$ and has for radius a uniform random variable $R(\theta)=$ $U(0.25,0.35)$. The Young modulus is taken equal to 1 and the poisson coefficient to zero. Classical FEM approaches use sampling techniques to solve this stochastic problem. For each sample, corresponding to an outcome of the geometry, a new mesh 
conforming to the geometry is created and an associated finite element computation is performed.

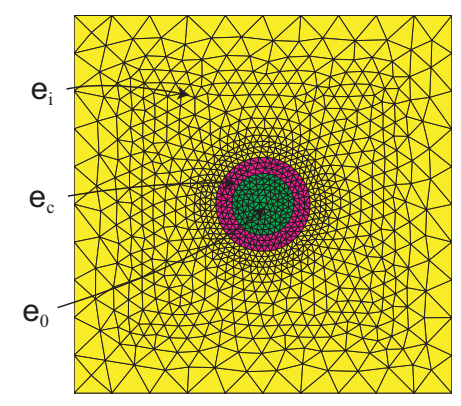

Figure 4. $X$-SFEM mesh and 3 element groups: surely in the domain $\left(e_{i}\right)$, possibly cut by the boundary of the hole $\left(e_{c}\right)$ and surely out the domain $\left(e_{o}\right)$

With the X-SFEM method proposed here, we build a unique mesh and we solve a unique system of Equation [12]. We use the mesh represented on Figure 4. This mesh is obtained by meshing a plate with a hole of radius $R=0.3$ (mean radius) and by meshing also the interior of this hole. It is convenient for comparing our approach with a classical FEM technique. A totally unstructured mesh could also have been used. Nevertheless, creating a mesh based on outcomes of the geometry allows to better control the mesh size around zones of interest and also leads to a higher regularity of the approximate solution at the stochastic level (see Section 4.6). On Figure 4, elements are split into three groups: elements surely in the domain, element possibly cut by the boundary of the hole and elements surely out the domain. We don't associate degrees of freedom with nodes belonging to elements of the third group and not belonging to elements of the first two groups. At the stochastic level, we use a finite element approximation with piecewise polynomials of degree $p=2$ (see Section 4.2.2): the mesh of $\boldsymbol{\Theta}=] 0.25,0.35\left[\right.$ that we use is $\left.\mathcal{T}_{k}=\right] 0.2500,0.2903,0.3321,0.3500[$. This mesh is obtained by an automatic procedure which will be detailed in a following paper. It is useful for numerical integration [20] and it allows one to obtain a good regularity per element of the approximate solution.

Figures 5 and 6 show a compararison of FEM and X-SFEM solutions for outcomes of the geometry. Corresponding outcomes of the iso-zero of the level set representing the hole are shown on the right figures. On Figure 7, we compare the response surface of the horizontal elongation $\Delta u(R)=u\left(R, x_{b}\right)-u\left(R, x_{a}\right)$, where $x_{a}=(0,1.5)$ and $x_{b}=(3,1.5)$. We note that X-SFEM leads to a very high precision solution within the whole range of variation of $R$. We must also note that with X-SFEM, problem [12] being solved, the solution obtained has an explicit form in terms of $R(\theta)$. Postprocessing can then be performed at a very low cost. An example of post-processing is illustrated on Figure 8 where the probability density function of $\Delta u$ is plotted. 

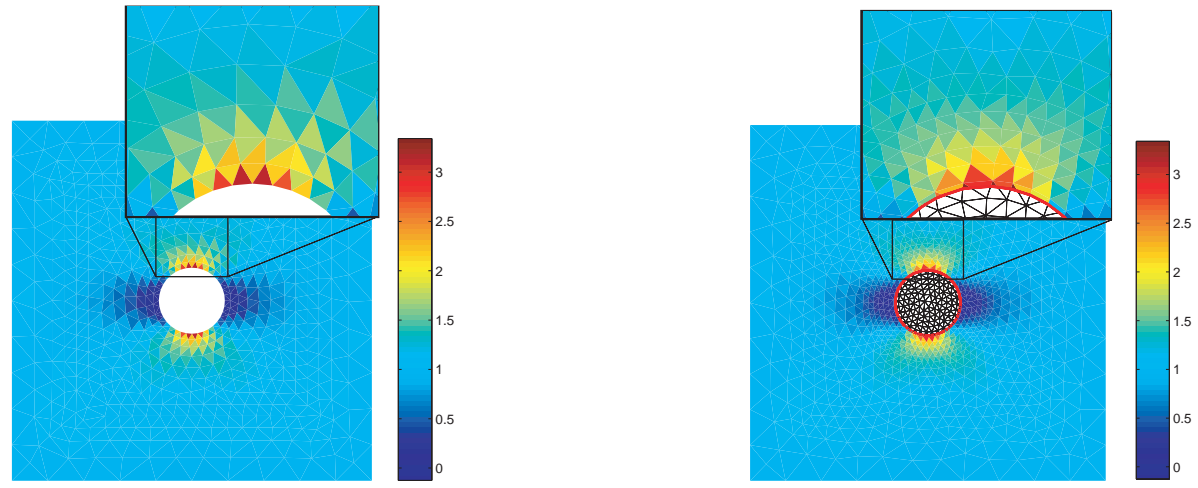

Figure 5. FEM (left) and X-SFEM (right) solutions: stresses $\sigma_{x x}$ for $R=0.275$
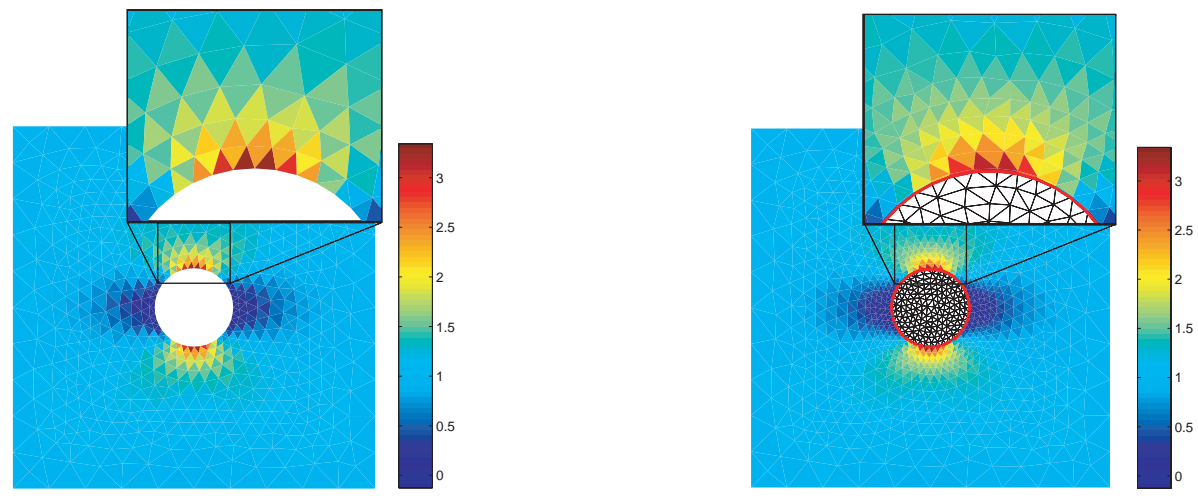

Figure 6. FEM (left) and X-SFEM (right) solutions: stresses $\sigma_{x x}$ for $R=0.325$

\section{Conclusion}

We proposed a stochastic finite element method based on X-FEM to solve stochastic partial differential equations defined on a random domain without remeshings. The random geometry is described in an implicit manner as the iso-zero of random level sets. A Galerkin approximation technique is then introduced which leads to a high precision approximate solution. At the stochastic level, a suitable finite element discretization is used which takes into account the lack of regularity of the approximate solution. It allows in some particular cases to obtain the exact solution of the stochastic problem and could allow in the general case to reach higher $p$ convergence rates than with classical spectral approximation such as polynomial chaos. Of course, the strategy proposed here is well adapted for parametric studies, which can be viewed as a particular case of a probabilistic study. 


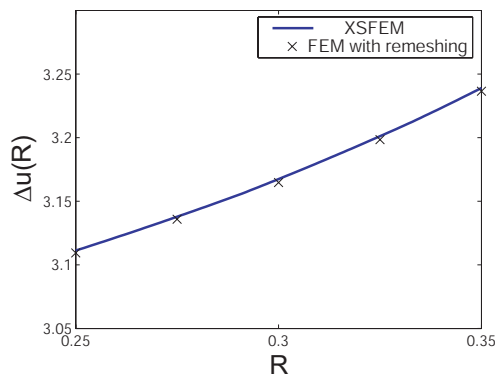

Figure 7. Comparison between FEM and $X$-SFEM: response surface $\Delta u(R)$

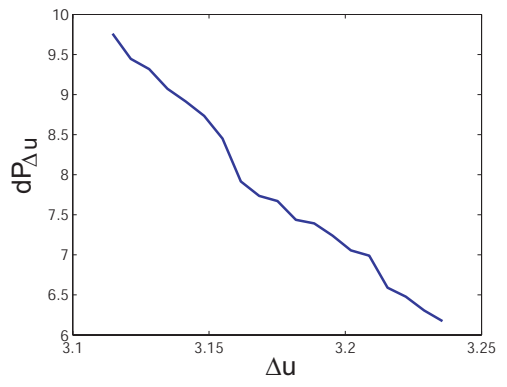

Figure 8. Probability density function of $\triangle u$ as a post-processing of $X-S F E M$

\section{Acknowledgements}

This work is supported by the French National Research Agency (ANR).

\section{References}

Babuska I., "Effects of uncertainties in the domain on the solution of Dirichlet boundary value problems", Numer. Math., vol. 93, n 4, 2003, p. 583-610.

Babuska I., Chleboun J., "Effects of uncertainties in the domain on the solution of Neumann boundary value problems in two spatial dimensions", Mathematics of Computation, vol. 71, $n^{\circ} 240,2002$, p. 1339-1370.

Babuska I., Tempone R., Zouraris G. E., "Solving elliptic boundary value problems with uncertain coefficients by the finite element method: the stochastic formulation", Computer Methods in Applied Mechanics and Engineering, vol. 194, 2005, p. 1251-1294.

Daux C., Moës N., Dolbow J., Sukumar N., Belytschko T., "Arbitrary branched and intersecting cracks with the eXtended Finite Element Method", Int. J. for Numerical Methods in Engineering, vol. 48, 2000, p. 1741-1760.

Deb M., Babuska I., Oden J., "Solution of stochastic partial differential equations using Galerkin finite element techniques", Computer Methods in Applied Mechanics and Engineering, vol. 190, 2001, p. 6359-6372.

Dolbow J., Moës N., Belytschko T., "Discontinuous enrichment in finite elements with a partition of unity method", Finite Elements in Analysis and Design, vol. 36, n 3-4, 2000, p. 235-260.

Ghanem R., "Ingredients for a general purpose stochastic finite elements implementation", Computer Methods in Applied Mechanics and Engineering, vol. 168, 1999, p. 19-34.

Ghanem R. G., Kruger R. M., "Numerical solution of spectral stochastic finite element systems", Comp. Meth. App. Mech. Eng., vol. 129, 1996, p. 289-303.

Ghanem R., Spanos P., Stochastic finite elements: a spectral approach, Springer, Berlin, 1991. 
Keese A., Mathhies H. G., "Hierarchical parallelisation for the solution of stochastic finite element equations", Computer Methods in Applied Mechanics and Engineering, vol. 83, 2005, p. 1033-1047.

Le Maître O. P., Knio O. M., Najm H. N., Ghanem R. G., "Uncertainty propagation using Wiener-Haar expansions", Journal of Computational Physics, vol. 197, 2004, p. 28-57.

Matthies H. G., Keese A., "Galerkin methods for linear and nonlinear elliptic stochastic partial differential equations", Computer Methods in Applied Mechanics and Engineering, vol.194, $\mathrm{n}^{\circ}$ 12-16, 2005, p. 1295-1331.

Melenk J. M., Babuska I., "The partition of unity method: basic theory and applications", Computer Methods in Applied Mechanics and Engineering, vol. 39, 1996, p. 289-314.

Moës N., Dolbow J., Belytschko T., "A finite element method for crack growth without remeshing”, Int. J. for Numerical Methods in Engineering, vol. 46, 1999, p. 131-150.

Nouy A., Schoefs F., «Technique de décomposition spectrale optimale pour la résolution d'équations aux dérivées partielles stochastiques », Proceedings of the Congrès Français de Mécanique (CFM 2005), Troyes, France, 2005. CD-ROM. http://belz.univubs.fr/lg2m/Documentation/CFM2005/articles/697.pdf.

Pellissetti M. F., Ghanem R. G., "Iterative solution of systems of linear equations arising in the context of stochastic finite elements", Advances in Engineering Software, vol. 31, 2000, p. 607-616.

Sethian J., Level Set Methods and Fast Marching Methods: Evolving Interfaces in Computational Geometry, Fluid Mechanics, Computer Vision, and Materials Science, Cambridge University Press, Cambridge, UK, 1999.

Soize C., Ghanem R., "Physical systems with random uncertainties: chaos representations with arbitrary probability measure", SIAM J. Sci. Comput., vol. 26, n² 2, 2004, p. 395-410.

Sukumar N., Chopp D., Moës N., Belytschko T., "Modeling holes and inclusions by level sets in the extended finite-element method", Computer Methods in Applied Mechanics and Engineering, vol. 190, 2001, p. 6183-6200.

Wiener N., "The homogeneous chaos", Am. J. Math., vol. 60, 1938, p. 897-936.

Xiu D. B., Karniadakis G. E., "The Wiener-Askey Polynomial Chaos for stochastic differential equations”, SIAM J. Sci. Comput., vol. 24, n² 2, 2002, p. 619-644. 